\title{
Influence of gain settings on strain ratios of elastographic image and texture parameters of B-mode image on thyroid tissue.
}

\author{
Mahdi Al-Qahtani ${ }^{1}$, Ravish Javed ${ }^{1}$, Ramsha Urooj Baig ${ }^{2}$, Eraj Humayun Mirza ${ }^{1,3 *}$ \\ ${ }^{1}$ Department of Biomedical Technology, College of Applied Medical Sciences, King Saud University, Riyadh, Kingdom \\ of Saudi Arabia \\ ${ }^{2}$ Department of Forensic Medicine and Toxicology, Dow University of Health Sciences, Karachi, Pakistan \\ ${ }^{3}$ Department Biomedical Engineering, Sir Syed University of Engineering and Technology, Karachi, Pakistan
}

\begin{abstract}
Thyroid nodules are benign though suspicious. Examination through elastography technique is beneficial as it provides real time and first hand evidence about the tissue stiffness by providing a strain value on comparing suspected and normal tissue. Until now, there is no established method that allows to categorise strain ratios whether tumour or normal. Changes in gain settings can significantly alter measurement and ultimately the clinical findings. The objectives of this study are to assess the effect of gain settings on strain ratios of isoechoic stiff lesions in a thyroid gland phantom, and the difference in strain ratios with first order texture parameters at different gain settings.

In this study we have reported the effect of gain setting parameter on strain ratios of the thyroid tumour. Furthermore, first order texture parameters (mean and variance) were discussed with respect to increase in gain settings.

Higher strain ratios were observed as gain increased. Significant differences in first order texture parameters can be observed for mean of tumour when compared with normal thyroid tissue. Variance did not demonstrate any significant difference.

It was concluded that gain parameter effect the strain ratios and texture parameters. This may affect the interpretation of results. It is advised to use elastography in combination with B-mode ultrasound, and sonographers must use a balanced approach when changing equipment parameters.
\end{abstract}

Keywords: Ultrasound, Elastography, Thyroid, Tumour, Texture analysis, Gain setting.

Accepted on January 15, 2018

\section{Introduction}

Nodule in a thyroid gland is considered to be abnormal [1]. This is a result of irregular cell growth within the thyroid gland $[2,3]$. Normally thyroid nodules can be determined via simple Ultrasonography (US) [4]. Conventional US have been used widely to distinguish the nodules to be biopsied. Although conventional US have shown to predict various pathological conditions accurately, but there are also significant number of cases that are contradicting results and variations in diagnostic performances of US that distinctly emanate the need to question the accuracy of the findings [5-7].

Physical examination of thyroid may lead to the prediction of a thyroid malignancy [4]. Healthy thyroid gland is commonly associated in the prognosis for uncovering the hardness, abnormality or malignancy of the gland. However, the technique of palpation is marred by its limitations; as it is subjective and it is difficult to determine deep pathological condition [8]. A relatively new technique; Ultrasound Elastography (UE) can be helpful in providing real-time evaluation of tissue stiffness in a particular Region of Interest (ROI) $[9,10]$.

UE provides a combination of B-mode ultrasound image and elastographic image which is a measure of strain ratio. Though, UE examination provides both the qualitative and quantitative diagnostic information; but, its accuracy and reproducibility are major concern for the clinicians. Clinicians use this combination of images to determine the extent of pathologic condition. However, this interpretation is subjective and dependent upon ability and experience of the observer $[11,12]$. Even though substantial years of experience and diagnostic capability of user/observer can fall out short in concluding the accurate pathology of disease. The observer dependability raises the issue of reproducibility and accuracy in interpretation of images, which may directly impact clinical diagnostic outcome. This outcome may often be a misjudgement or misinterpretation due to various parameter settings in the equipment itself. Variation in ultrasound equipment parameters has demonstrated contradicting results over the time $[13,14]$. 
However, gain setting of ultrasound has shown to significantly alter measurements and eventually clinical findings [15].

Analysis of images by computed means is usually done to find even slight changes that cannot be distinguished via human eye [16]. Analysing the image texture is of vital importance as it allows better plan of action for the clinicians. In this study we report the effect of gain settings on strain ratios of isoechoic stiff lesions in a thyroid gland phantom. Furthermore, we have associated the difference in strain ratios with first order texture parameters at different gain settings while keeping rest of the parameter unchanged.

\section{Methodology}

In this study a tissue phantom was selected that contain all human body parameters. The rationale behind choosing a phantom was to elucidate the changes caused due to movement of patients, difference in patient body mass index, age, and other parameters that may affect image directly or indirectly. The current study was conducted at department of biomedical technology, college of applied medical sciences, King Saud University. All the examination was performed at room temperature. This is an experimental study to determine the change in various parameters associated with image due to change in ultrasound gain settings.

\section{Ultrasound examination}

B-Mode ultrasound examination was performed on thyroid gland phantom with isoechoic stiff lesion (Model 074. Thyroid Ultrasound training phantom, CIRS, Norfolk, Virginia 23513, USA) via SonixTouch Q+ (Analogic Corporation, 8 Centennial Drive, Peabody, MA 01960, USA) UE machine using a linear probe at a frequency of $10 \mathrm{MHz}$ with variable gains of $40 \%$, $50 \%$ and $60 \%$. All other parameters that might affect the ultrasound image interpretation were kept unchanged throughout the experiment (Frequency, depth, time gain compensation, brightness and contrast). The thyroid ultrasound examination was performed via previously described guidelines as per American Institute of Ultrasound in medicine [17].

\section{Elastographic strain ratio}

After examining the B-mode image, elastographic mode was selected to measure the strain ratios of the tumour. To determine strain ratios, Region of Interest (ROI) positioned within the tumour and outside the tumour (healthy surrounding tissue) within the thyroid gland was selected by using the free hand method as shown in Figure 1 [18].

\section{Image selection}

For each gain setting a total of 5 best images that displayed a good tumour boundary were selected from a pool of 99 images from a cine loop. In each image 3 random areas were selected to determine strain ratios.

\section{First order texture parameters}

To compare the texture parameters of thyroid tumour tissue and healthy thyroid tissue an ROI that included both the tumour and surrounding thyroid tissue was selected. Images were analysed using MAZDA software. ROI of 400 pixels in each site (tumour and normal tissue) was selected. Size of ROI was kept unchanged for all the images. 5 images at each gain setting were analysed for mean and variance.

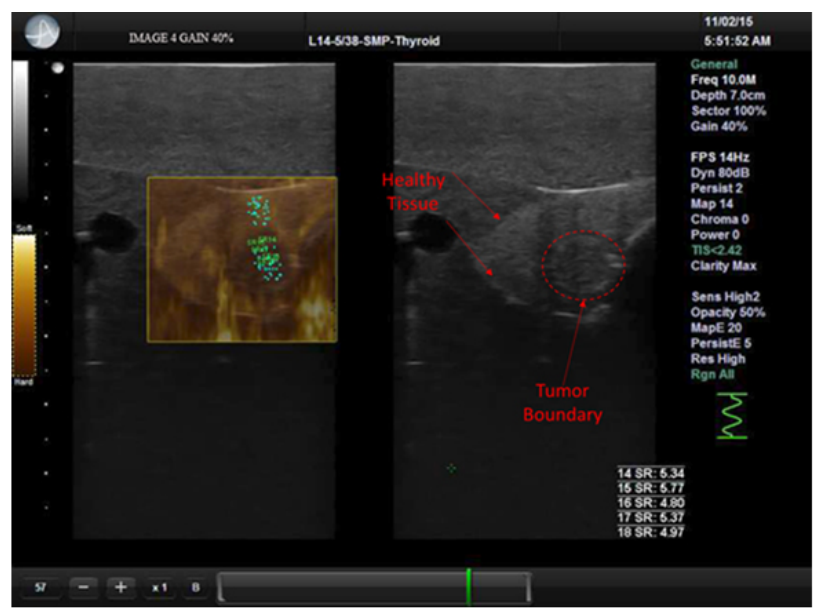

Figure 1. Elastographic image with B-mode ultrasound image, highlighting tumour and healthy tissue.

\section{Statistical analysis}

IBM SPSS Statistics v21 was used for statistical analysis. One way ANOVA was applied to determine the significant difference of strain ratios between the gain settings. A p-value of 0.05 was taken to be as significant difference. All the values are present as mean $\pm \mathrm{SD}$.

\section{Results}

Thyroid tissue when ascertained by elastographic examination manifested a drop off trend in strain ratios of normal tissue to tumour tissue when the gain is increased. Cumulative elastographic strain ratio scores are shown in Figure 2 (i). It was observed that even $10 \%$ increase in gain setting demonstrated a significant difference in strain ratio. Elastographic strain ratios for a gain of $40 \%$ averaged at $5.38 \pm$ 0.56 , while gains of $50 \%$ and $60 \%$ indicated the strain ratios to be $3.07 \pm 0.28$ and $2.23 \pm 0.34$ respectively.

First order textural analysis of mean and variance for normal and tumour tissues at different gain settings are presented in Figures 2 (ii) and (iii) respectively. Cumulative mean at different gain settings revealed significantly lowered mean values for tumour tissue when compared to normal thyroid tissue at each gain setting. However, the values of mean for both normal and tumour thyroid tissue increased with an increase in a gain (Figure 2 (ii)).

A variable trend was observed for variance values when normal thyroid tissue was compared with pathological tissue. This tendency was unlike the one seen in mean values. 
Although increasing the gain was directly proportional to variance values for both the normal and diseased tissue, nevertheless, there was no significant difference between the variance of normal and tumour tissue at each gain setting. Significant differences were observed for variance readings for tumour at each gain setting. However, the only significant difference for normal tissue was observed between gains of $40 \%$ and $60 \%$ (Figure 2 (iii)).

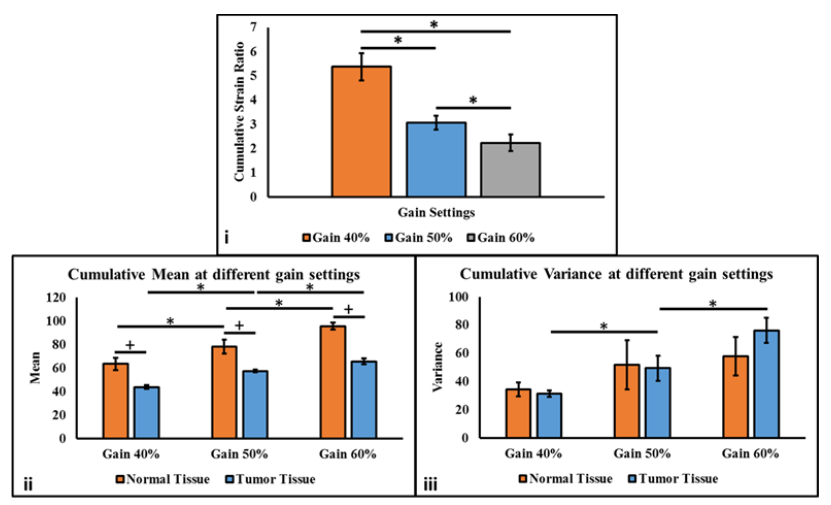

Figure 2. (i) Cumulative strain ratios of thyroid tumour with respect to normal thyroid tissue; (ii) Cumulative mean values of tumour and normal thyroid tissue at different gain settings; (iii) Cumulative variance values of tumour and normal thyroid tissue at different gain settings. ${ }^{+}$Represents significant difference between tumour types; ${ }^{*}$ Represents significant difference between gain settings.

Visual impact of change in gain was also obvious that can be seen via Figures 3 (i-iii). A gain of $60 \%$ demonstrates a good lower boundary while the upper boundary of tumour is fused with the surrounding tissue. Whereas, a gain setting of $40 \%$ and $50 \%$ demonstrates a distinguishable tumour boundary from the surrounding tissue.
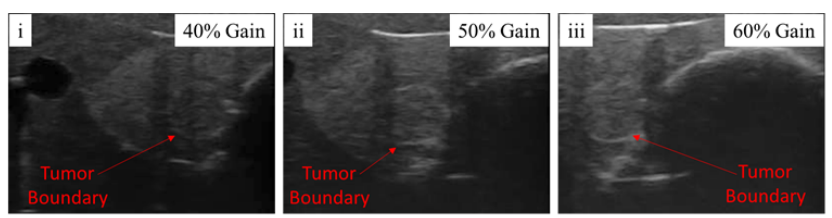

Figure 3. Ultrasound images of thyroid gland in a phantom displaying tumour boundaries at a gain setting of: (i) 40\%; (ii) $50 \%$; (iii) $60 \%$

\section{Discussion}

An increase in stiffness and inflexibility of a tissue is usually considered alarming [19]. Soft tissue and tissue with lesser stiffness tends to be healthy and have least symptoms to develop cancer or other adverse diseases [20-22]. Palpation technique is used since decades to detect stiffness changes in a tissue. Strain Elastography (SE) also works on the principle of palpation [23]. In elastography, the stiffness is determined by measuring the ratio stiffness between the two regions of a tissue. Various studies have been performed on breast, thyroid, liver and other tissues [10,24-27]. Unfortunately, even after countless research conducted and concluded on tissue stiffness; none of the studies are able to establish a standard to outline tissues as tumour and other as normal tissue (healthy tissue). To assess the impact of parameter change in elastographic strain ratios and its further impact on texture features of an image, we studied effect of changing the gain, because any change in gain affects the noise level of the image [28]. Our results reveal a significant difference among strain ratio values when the gain shifted even $10 \%$. It is suggested that strain ratios that are determined via elastography technique are dependent upon changing the equipment parameters. Previous studies have shown that gain settings effect the texture parameters but this is first study where we suggest that strain ratios of an elastographic image is also dependent upon gain change. Region of interest, pre-compression of tissue and elastography image scoring are also reported to effect the interpretation of results of elastographic image [29-31]. In a study it was reported that elastography is operator dependant and inter-observer variability in examination may result [32]. Figure 4 (i) shows elastographic strain ratios of individual slices in an ultrasound image.

From Figure 4 (i) it can be clearly deduced that although there were significant changes to elastographic strain ratios cumulatively but the strain ratios did not changed much for higher gain settings. On the other hand gain settings of $40 \%$ demonstrated higher strain ratios.

Extracting texture features of an image greatly enhance the accuracy and precision to characterise the image and it further helps the clinicians to make better decision and medical intervention.

Effect of gain settings have been reported previously on texture properties of the image in various studies [16,33]. However, there are no studies that determine the differences in texture parameter due to gain settings. Analysis of the first order texture features (mean and variance) by altering the gain settings on tumour and normal thyroid tissue, uncovered significant impact only on mean values for both tumour and normal thyroid tissue. However, no significant difference between the two tissue types was observed. Individual values of mean and variance in each slice of ultrasound B-mode image at different gain settings for tumour and normal thyroid tissue are shown in Figure 4 (ii (A-F)).

The reason for a significant change in mean values is because computerised analysis is an objective assessment, while in the clinical situation sonographers keep on changing the gain parameters with respect to every patient. Due to change in gain parameter, shadowing takes place and this eventually changes the texture of ultrasound image [14]. Authors have reported that first order statistics can be the discriminating feature in a tissue [34].

It is advised that a balance of gain and other parameters must be observed and a change in these parameters may result in variable interpretation that might risk the well-being of the patient. Furthermore, a range of parameters in the equipment itself must be researched and their impact on various tissues should be determined and a relation between these parameters must be established. In the current study elastographic strain 
ratios demonstrated more dependable results when the gain settings are changed. However among first order statistics, mean was the only variable on which to depend for characterisation of the tissue.

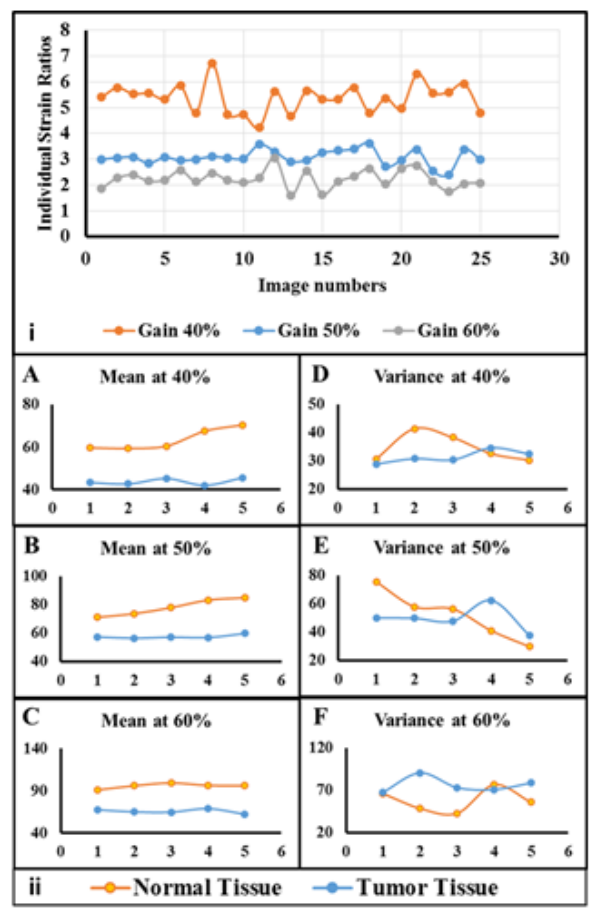

Figure 4. (i) Individual values of strain ratios of thyroid tumour with respect to normal thyroid tissue in each individual image slice; (ii) Individual values of mean $(A-C)$ and variance $(D-F)$ of thyroid tumour with respect to normal thyroid tissue in each individual image slice.

\section{Conclusion}

Equipment parameters play a major role in interpretation and judgement of the results. Gain parameter directly affects the strain ratios and texture features. Better plan of action is achievable when combing B-mode ultrasound and elastographic imaging modalities. Additional research is mandatory to establish a relationship between the equipment parameters and strain ratios. It is advised that sonographer adopt more balanced approach when changing the equipment parameters. Furthermore, values of mean can be taken as detrimental factor to differentiate tumour from healthy tissues.

\section{Acknowledgement}

All the studies were performed at department of Biomedical Technology, College of Applied Medical Sciences at King Saud University. The authors extend their appreciation to the College of Applied Medical Sciences Research Center and the Deanship of Scientific Research at King Saud University for funding this research. The authors would like to thank Dr. Alain Nchimi for his efforts in critically reviewing the manuscript.

\section{References}

1. Tan GH, Gharib H. Thyroid incidentalomas: management approaches to non-palpable nodules discovered incidentally on thyroid imaging. Ann Intern Med 1997; 126: 226-231.

2. Luo S, Kim E-H, Dighe M, Kim Y. Thyroid nodule classification using ultrasound elastography via linear discriminant analysis. Ultrasonics 2011; 51: 425-431.

3. Vidinov K, Kalniev M, Sechanov T. Is total thyroidectomy the optimal treatment for benign thyroid disease? Comptes rendus de 1 academie bulgare des sciences 2013; 66: 1057-1062.

4. Pacini F, Castagna M, Brilli L, Pentheroudakis G, Group EGW. Thyroid cancer: ESMO clinical practice guidelines for diagnosis, treatment and follow-up. Ann Oncol 2010; 21: 214-219.

5. Moon WJ, Baek JH, Jung SL, Kim DW, Kim EK, Kim JY. Ultrasonography and the ultrasound-based management of thyroid nodules: consensus statement and recommendations. Kor J Radiol 2011; 12: 1-14.

6. Association AT. Guidelines Taskforce on thyroid nodules and differentiated thyroid cancer. Revised American Thyroid Association management guidelines for patients with thyroid nodules and differentiated thyroid cancer. Thyroid 2009; 19: 1167-1214.

7. Gharib H, Papini E, Paschke R, Duick D, Valcavi R, Hegedüs L. AACE/ACE/AME European Thyroid Association medical guidelines for clinical practice for the diagnosis and management of thyroid nodules. Endocr Pract 2010; 16: 1-43.

8. Park SH, Kim SJ, Kim E-K, Kim MJ, Son EJ, Kwak JY. Interobserver agreement in assessing the sonographic and elastographic features of malignant thyroid nodules. Am J Roentgenol 2009; 193: 416-423.

9. Al-Qahtani M. Shear-wave and strain elastography: A Comparative review on principles, basic techniques and applications. Curr Med Imaging Rev 2016; 12: 269-278.

10. Al-Qahtani M, Mirza EH, Al-Qahtani MD, Al-Muqati MF. A comparative study of shear-wave elastography and strain elastography on a breast phantom for diagnosis of tumor and cyst. J Biomed Eng Med Imaging 2015; 2: 24

11. Huang YL, Chen DR, Jiang YR, Kuo SJ, Wu HK, Moon W. Computer-aided diagnosis using morphological features for classifying breast lesions on ultrasound. Ultrasound Obstetr Gynecol 2008; 32: 565-572.

12. Wolstenhulme S, Davies A, Keeble C, Moore S, Evans J. Agreement between objective and subjective assessment of image quality in ultrasound abdominal aortic aneurism screening. Brit J Radiol 2015; 88: 20140482.

13. Peters SA, Bots ML, Lind L, Groenewegen KA, de Korte $\mathrm{C}$, den Ruijter HM. The impact of variability in ultrasound settings on the measured echolucency of the carotid intimamedia. J Hypertension. 2013; 31: 1861-1867.

14. Sivaramakrishna R, Powell KA, Lieber ML, Chilcote WA, Shekhar R. Texture analysis of lesions in breast ultrasound 
images. Computerized Med Imaging Graphics 2002; 26: 303-307.

15. Potter K, Reed CJ, Green DJ, Hankey GJ, Arnolda LF. Ultrasound settings significantly alter arterial lumen and wall thickness measurements. Cardiovas Ultrasound 2008; 6: 1-11.

16. Smutek D, Šára R, Sucharda P, Tjahjadi T, Švec M. Image texture analysis of sonograms in chronic inflammations of thyroid gland. Ultrasound Med Biol 2003; 29: 1531-1543.

17. Medicine AIoUi. AIUM Practice guideline for the performance of thyroid and parathyroid ultrasound examination. J Ultrasound Med 2003; 22: 1126.

18. Öztürk M, Yildirim R. The usefulness of strain wave elastography in the diagnosis and grading of Hashimoto's thyroiditis in children. La radiologia medica 2017; 122: 960-966.

19. Uliaque CF, Berdún FP, Herrero RL, Lórenz CP. Usefulness of semi-quantitative elastography in predicting malignancy in thyroid nodules. Radiología 2016; 58: 366-372.

20. Garra BS. Imaging and estimation of tissue elasticity by ultrasound. Ultrasound Quarterly 2007; 23: 255-268.

21. Hong Y, Liu X, Li Z, Zhang X, Chen M, Luo Z. Real-time ultrasound elastography in the differential diagnosis of benign and malignant thyroid nodules. J Ultrasound Med 2009; 28: 861-867.

22. Xie P, Xiao Y, Liu F. Real-time ultrasound elastography in the diagnosis and differential diagnosis of sub-acute thyroiditis. J Clin Ultrasound 2011; 39: 435-440.

23. Bamber J, Cosgrove D, Dietrich C, Fromageau J, Bojunga J, Calliada F. EFSUMB guidelines and recommendations on the clinical use of ultrasound elastography. Part 1: Basic principles and technology. Eur J Ultrasound 2013; 34: 169-184.

24. Monpeyssen H, Tramalloni J, Poirée S, Hélénon O, Correas JM. Elastography of the thyroid. Diagnostic and Interventional Imaging. 2013;94(5):535-44.

25. Rykhtik P, Ryabova E, Vasenin S, Shkalova L, Zagaynov V. Experience of using elastography in the diagnosis of liver fibrosis in the practice of hepatology center. Radiology 2015; 1: 49 .
26. Nicolas E, Callé S, Remenieras J-P. Generating Shear waves in the human brain for ultrasound elastography: A new approach. Phys Procedia 2015; 70: 1255-1259.

27. Uramoto $H$, Nakajima $Y$, Ohtaki $K$, Kinoshita $H$. Intraoperative ultrasound elastography has little diagnostic benefit for deeper tumours of the lung. Eur J Cardio-Thorac Surg 2015; ezv340.

28. Chan K. Adaptation of ultrasound image texture characterization parameters. Eng Med Biol Soc 1998.

29. Barr RG. Sonographic breast elastography. J Ultrasound Med 2012; 31: 773-783.

30. Lyshchik A, Higashi T, Asato R, Tanaka S, Ito J, Mai JJ. Thyroid gland tumor diagnosis at US elastography. Radiology 2005; 237: 202-211.

31. Kamoi K, Okihara K, Ochiai A, Ukimura O, Mizutani Y, Kawauchi A. The utility of transrectal real-time elastography in the diagnosis of prostate cancer. Ultrasound Med Biol 2008; 34: 1025-1032.

32. Fleury E, Fleury J, Piato S, Roveda Jr D. New elastographic classification of breast lesions during and after compression. Diagn Interv Radiol 2009; 15: 96-103.

33. Wu JY, Beland M, Konrad J, Tuomi A, Glidden D, Grand D. Quantitative ultrasound texture analysis for clinical decision making support. SPIE Med Imaging 2015.

34. Layer G, Zuna I, Lorenz A, Zerban H, Haberkorn U, Bannasch P. Computerized ultrasound B-scan texture analysis of experimental diffuse parenchymal liver disease: Correlation with histopathology and tissue composition. J Clin Ultrasound 1991; 19: 193-201.

\section{*Correspondence to}

Eraj Humayun Mirza

Department Biomedical Engineering

Sir Syed University of Engineering and Technology

Karachi

Pakistan 